However, I thank you very much for the kind reception you have given me.

On motion, the meeting adjourned at 10.45 P. M.

[Communicated after Adjournment by Edward P. ThompSON.]

Having followed the invention underlying Prof. Matthews' new photometer, through the United States and foreign Patent Offices, and having been unable to attend the meeting, I take pleasure in adding my commendation of the valuable experimental and practical work obtained by the author of the paper. It may not be amiss to add something about the actual novelty of the device considered as an invention. This may best be accomplished by quoting one of the claims granted by the United States Patent Office as an award for his ingenuity.

"In an apparatus for measuring candle power, the combination with the support for the light to be tested, of a photometer screen, and mirrors so fixed as to illuminate said screen proportionally to the mean spherical candle power of said light, said mirrors remaining stationary during the operation of measuring the light."

This is one of the twenty-three claims granted by the Patent Office. It serves to show that Prof. Matthews has originated a new type of photometer, and the members will no doubt greatly appreciate his services in providing an instrument which will so readily save time and tedious calculation.

I have also examined complete drawings of Mr. Burnett's photometer for measuring illumination in terms of the number of luxes instead of in terms of distance. The device as practially carried out is exceedingly simple, and without movable parts. There is nothing to get out of order.

\title{
CRITICISM ON THE MEAN SPHERICAL CANDLE POWER AS A STANDARD FOR ILLUMINATION.
}

[Communicated after Discussion by Alexander J. Wurts.]

The mean spherical candle power is unquestionably the present accepted standard of light, but in reviewing a subject of this kind, it often is instructive to consider whether we may not be somewhat biased by a practice, rule or standard which has ceased to be either useful or practical. The question arises as to whether we wish to continue this standard as our guide. Does the so-called standard meet with our approval? Does the mean spherical candle power, as a standard, measure that which we desire to have measured? In the opinion of the writer, it fails to do so and it would seem that something which more nearly meets our present needs should be devised.

In years gone by, when the incandescent lamp was practically the only electric lamp in commercial use, and later, even after the arc lamp had been introduced, the photometric determination 